\title{
A LOW-ACCELERATION MEASUREMENT USING ANTI-VIBRATION TABLE WITH LOW-FREQUENCY RESONANCE
}

\author{
T. Shimoda ${ }^{1}$, W. Kokuyama ${ }^{2}$, H. Nozato ${ }^{3}$ \\ ${ }^{1}$ National Metrology Institute of Japan (NMIJ/AIST), Tsukuba, Japan, tomofumi.shimoda@aist.go.jp \\ ${ }^{2}$ National Metrology Institute of Japan (NMIJ/AIST), Tsukuba, Japan, wataru.kokuyama@aist.go,jp \\ ${ }^{3}$ National Metrology Institute of Japan (NMIJ/AIST), Tsukuba, Japan, hideaki.nozato@aist.go.jp
}

\begin{abstract}
:
This manuscript describes how NMIJ isolates interferometer optics from the ground vibration for low-acceleration measurement by installing an antivibration table. Such a vibration isolation system is designed for an accelerometer calibration system to reduce vibration noise from the microtremor or from reaction of the vibration exciter. Mitigating the vibration of optics enables evaluation of accelerometers at small amplitudes, which is required in aerospace or infrastructure monitoring applications. In this manuscript, vibration transmissibility of the anti-vibration table is measured using a triaxial seismometer, and its benefit in the calibration system is discussed.
\end{abstract}

Keywords: Laser interferometer, anti-vibration table, microtremor, ground noise, low-acceleration measurement

\section{INTRODUCTION}

Low-acceleration measurements are gradually required from the viewpoint of various industrial fields such as aerospace or infrastructure monitoring. The resolution of earth observation by satellite imaging, which is one of the main aerospace applications, is suffering from micro-vibrations [1]. In an on-orbit satellite, moving instruments such as mechanical gyroscopes or reaction wheels can generate micro-vibration, which is typically smaller than $10^{-2} \mathrm{~m} / \mathrm{s}^{2}$ [2]. In infrastructure monitoring, continuous measurement of eigenfrequency of structure using microtremor, which is in the order of $10^{-3} \mathrm{~m} / \mathrm{s}^{2}$ or less, is proposed $[4,5]$. The demand for such measurements is gradually increasing with the aging of much of the infrastructure.

As the basis for these applications, evaluation of accelerometer is essential to verify the reliability of measurements. A calibration system using a laser interferometer and a vibration exciter is used to determine the response of an accelerometer [6]. In this system, a target accelerometer is vibrated by the shaker, and its displacement is precisely monitored by the interferometer for calibration. For lowacceleration, the calibration result can suffer from the background noise of the interferometer, which originates from seismic vibration, self-noise of the interferometer, and so on. Mitigation of such noise is necessary to meet the demands of lowacceleration measurements.

For these purposes, we try to reduce the noise from microtremor, which typically appears below a few hundred Hz. As the first step, it is evaluated how to reduce the microtremor noise in the lowfrequency calibration system in NMIJ [7]. Overview of the microtremor issue and schematics of noise reduction are presented in Section 2. Next, in Section 3, experimental results using an antivibration table with a low resonant frequency are reported. The conclusion is described in Section 4.

\section{SEISMIC NOISE IN A CALIBRATION SYSTEM}

\subsection{Overview of Accelerometer Calibration}

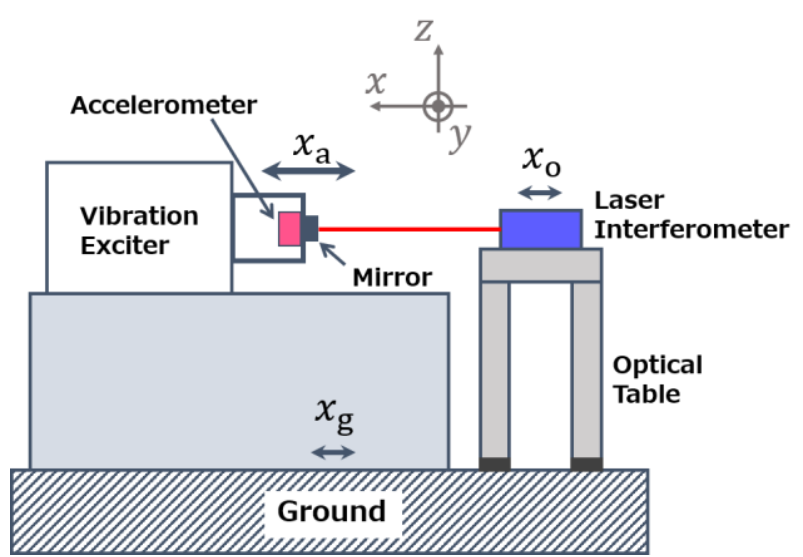

Figure 1: Schematics of a low-frequency accelerometer calibration system.

Figure 1 shows the schematics of the lowfrequency calibration system. A target accelerometer is fixed to a vibration exciter and vibrated at a certain frequency and amplitude. At the same time, the displacement of the accelerometer is measured by a laser interferometer constructed on 
another table. The output signal from the accelerometer is then compared to the measured displacement to evaluate the sensitivity.

In this process, the accelerometer responds the absolute vibration $x_{\mathrm{a}}$ with respect to the inertial frame, while the laser interferometer measures the relative vibration $\left(x_{\mathrm{a}}-x_{\mathrm{o}}\right)$ between the optical table and the reflection surface on the exciter. These two vibrations differ by the vibration of the optical table $x_{0}$, which originates from the reaction of the shaker or the microtremor;

$$
x_{\mathrm{o}}=r H_{\mathrm{o}} x_{\mathrm{a}}+H_{\mathrm{o}} x_{\mathrm{g}},
$$

where $r$ denotes the fraction of the reaction, $H_{\mathrm{o}}$ the vibration transmissibility of the optical table, and $x_{\mathrm{g}}$ the microtremor. If the reaction is carefully suppressed, this discrepancy is not important in usual cases because the vibration amplitude of the shaker is sufficiently larger than that of the optical table induced by the microtremor; $x_{\mathrm{a}} \gg H_{\mathrm{o}} x_{\mathrm{g}}$. However, the effect of the microtremor becomes relatively non-negligible in the low-acceleration measurement mentioned in Section 369. Figure 2 presents the amplitude spectral density (ASD) of the optical table vibration $x_{0}$ of the current system induced by the microtremor, measured with a broadband triaxial seismometer (Trillium Compact 120s) in NMIJ. The vibration is on the order of $10^{-4}-10^{-6} \mathrm{~m} / \mathrm{s}^{2} / \sqrt{\mathrm{Hz}}$.

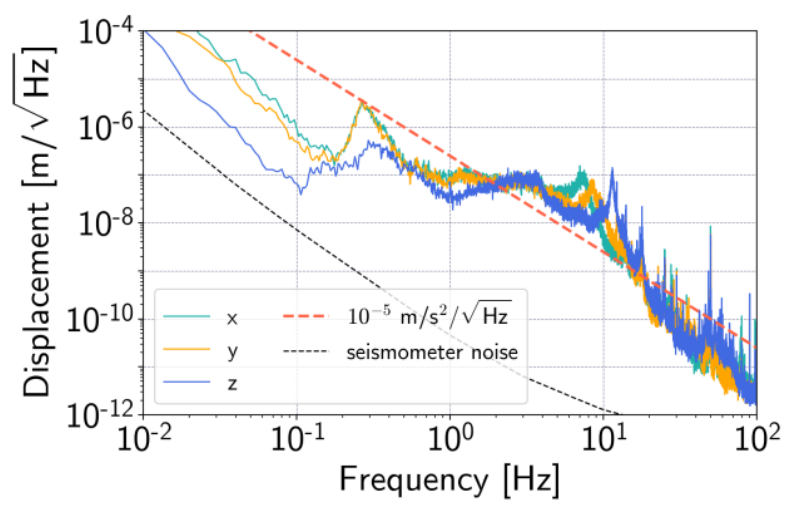

Figure 2: ASD of the optical table vibration in $\mathrm{x}$ (green), $\mathrm{y}$ (orange), and $\mathrm{z}$ (blue) directions, which was measured in NMIJ, Japan. Self-noise of the seismometer is plotted with the dashed black line. The dashed red line shows $10^{-5} \mathrm{~m} / \mathrm{s}^{2} / \sqrt{\mathrm{Hz}}$ for comparison.

Figure 3 shows the ASDs of the interferometer signal measuring $\left(x_{\mathrm{a}}-x_{\mathrm{o}}\right)$ of the low-frequency calibration system and of the seismometer signal measuring $x_{0}$, when the vibration exciter is turned off. Sum of these two signals corresponds to the noise in determining $x_{\mathrm{a}}$. The vibration of the optical table dominates the total noise below $10 \mathrm{~Hz}$. Therefore, suppression of vibration is one of the primary requirements for accelerometer calibration at small acceleration amplitudes.

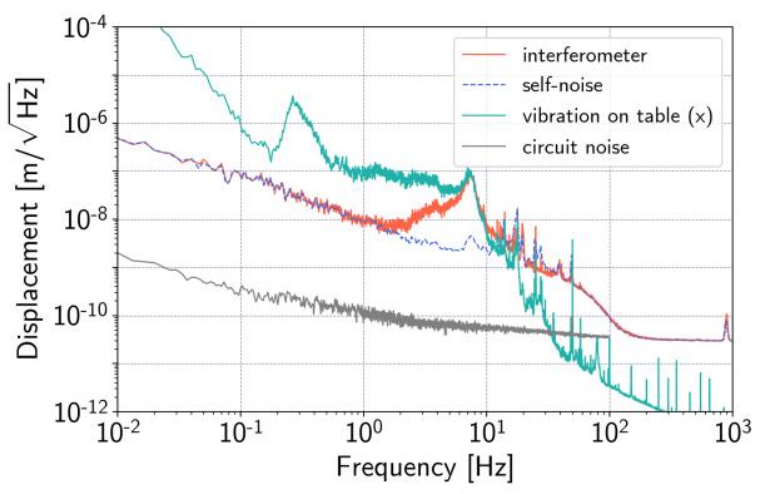

Figure 3: ASD of the interferometer signal of the lowfrequency calibration system (red), circuit noise of the interferometer (grey), and the optical table vibration (green). The dashed blue line shows the self-noise of the interferometer, which was estimated by subtracting the table vibration from the interferometer signal.

Additionally, signal from other than vibration, which is plotted with dashed blue line in Figure 3, limits the current performance above $10 \mathrm{~Hz}$. Cyclic error of the interferometer, which originates from non-linearity of the signal, is a suspicious noise source between $10 \mathrm{~Hz}$ and $100 \mathrm{~Hz}$. The electrical circuit noise is dominant above $100 \mathrm{~Hz}$. Mitigation of such noise sources is also essential to improve the overall performance. To determine $10^{-3} \mathrm{~m} / \mathrm{s}^{2}$ amplitude for calibration with $1 \%$ accuracy in 1 second, the ASD of the background noise needs to be below $10^{-5} \mathrm{~m} / \mathrm{s}^{2} / \sqrt{\mathrm{Hz}}$ at the oscillation frequency. In this manuscript, we focus on the reduction of the vibration noise.

\subsection{Low-frequency Vibration Isolation}

It is straightforward to reduce the vibration transmissibility $H_{\mathrm{o}}$ for low-acceleration measurement. $H_{\mathrm{o}}$ of a single mass-spring-damper model has a form of

$$
H_{\mathrm{o}}(f)=\frac{f_{0}^{2}}{f_{0}^{2}+i f f_{0} / Q-f^{2}},
$$

which is characterized by the resonant frequency $f_{0}$ and the quality factor $Q$. As the equation shows, vibration is suppressed above the resonant frequency in proportional to $f^{-2}$ in an ideally simple system. The current table has $f_{0} \sim 7 \mathrm{~Hz}$ and $Q \sim 10$. In this case, the microtremor is not sufficiently isolated below $10 \mathrm{~Hz}$, where the vibration noise is dominant. Low resonant frequency below $1 \mathrm{~Hz}$ is desired to suppress the excess of the vibration in Figure 2 down to $1 \mathrm{~Hz}$.

To achieve the low resonant frequency, we are installing an anti-vibration system, which has resonant frequencies of $0.25 \mathrm{~Hz}$ in horizontal and vertical directions. The system consists of a springantispring system, in which the restoring force of the suspension is partially cancelled by the antirestoring force from gravity or the elastic part. This enables the low resonant frequency with relatively 
small size $(\sim 0.7 \mathrm{~m})$. For comparison, a simple pendulum-type isolation system requires $4 \mathrm{~m}$ size to achieve $0.25 \mathrm{~Hz}$. The optical table in Figure 1 is replaced with the low-frequency anti-vibration system shown in Figure 4.

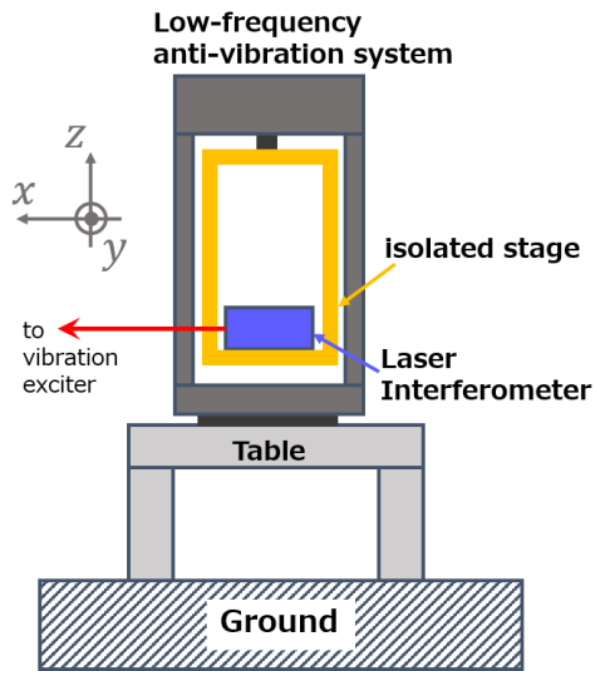

Figure 4: Schematic diagram of the low-frequency antivibration system for the laser interferometer.

As Equation () shows, suppression of the transmissibility also contributes to the reduction of the reaction from vibration excitation through the ground vibration. However, it should be noted that response to external force fluctuation such as sound or airflow can be enhanced below the resonant frequency, because the low-frequency system has low stiffness. Excess of low-frequency fluctuation induces alignment fluctuation, which affects the calibration result at higher frequencies. Therefore, the environmental disturbance is also an important factor that determines the performance.

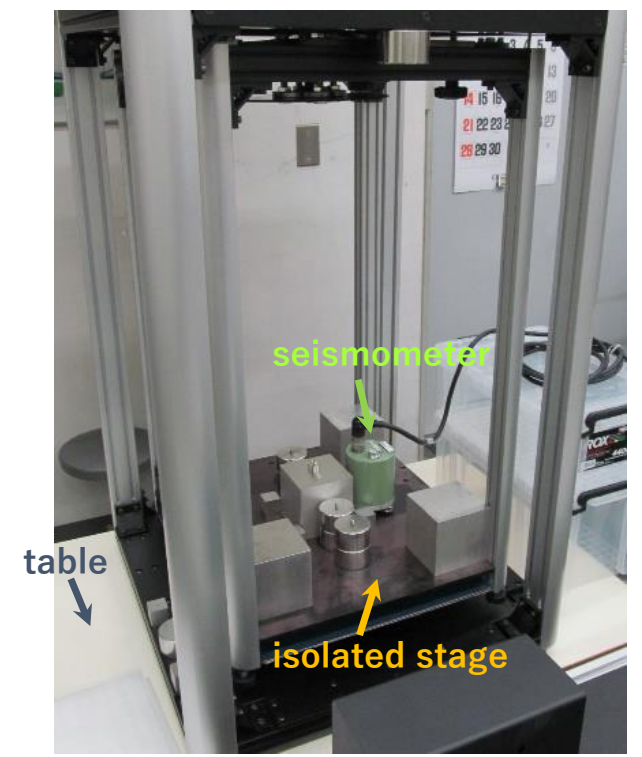

Figure 5: Vibration measurement on the anti-vibration stage.

\section{EVALUATION OF AN ANTI-VIBRATION SYSTEM}

\subsection{Vibration Transmissibility Measurement}

In order to evaluate the performance of the system, a seismometer was placed on the isolated stage as shown in Figure 5.

After the vibration measurement on the stage, the seismometer was then moved to the table where the anti-vibration system is placed. The vibration spectrums at these points were compared to estimate the vibration transmissibility of the system.

Measured vibration spectrums are presented in Figure 6 . The vibration on the isolated stage was almost as expected from the vibration on the table (blue line) and the theoretical vibration transmissibility (equation (2)). Here a resonant frequency $f_{0}=0.3 \mathrm{~Hz}$ and a quality factor $Q=1$ were assumed. The microtremor is successfully suppressed by $\sim 100$ times around a few $\mathrm{Hz}$, and the residual noise level is below $10^{-5} \mathrm{~m} / \mathrm{s}^{2} / \sqrt{\mathrm{Hz}}$ above $1 \mathrm{~Hz}$ except for the peak at $7 \mathrm{~Hz}$, though the seismometer signal was not measured correctly above $20 \mathrm{~Hz}$ because of the data logger noise.

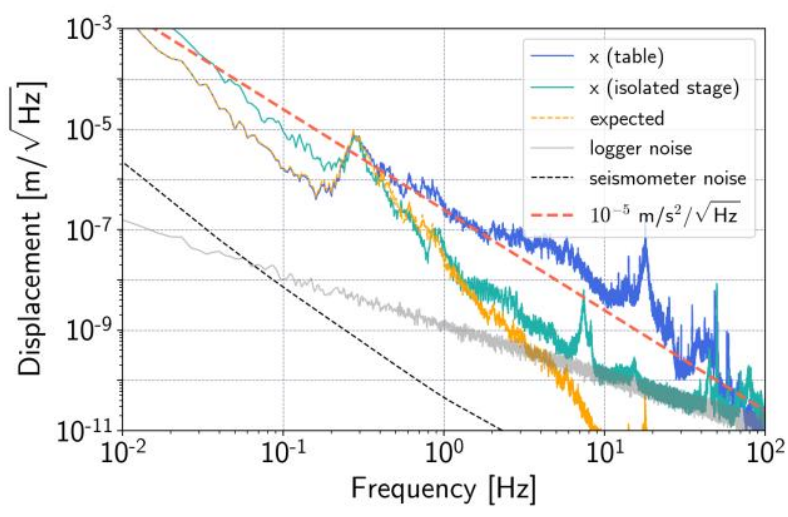

Figure 6: Performance of the anti-vibration system. The blue and green lines show the spectrum on the table and on the isolated stage, respectively. The orange line is expected spectrum on the stage. The black dashed and grey lines are the self-noise of the seismometer and the data logger, respectively.

On the other hand, vibration below $0.2 \mathrm{~Hz}$ was increased on the isolated stage, by about ten times than on the table. Such excess may originate from external force disturbances, because the antivibration system has low stiffness to lower the resonant frequency, as mentioned in Section 2.2. A wind shield is planned to be installed around the anti-vibration system to protect it from the airflow or sound. The other possible disturbances, such as tilt fluctuation of the stage or temperature fluctuation, should also be evaluated. 


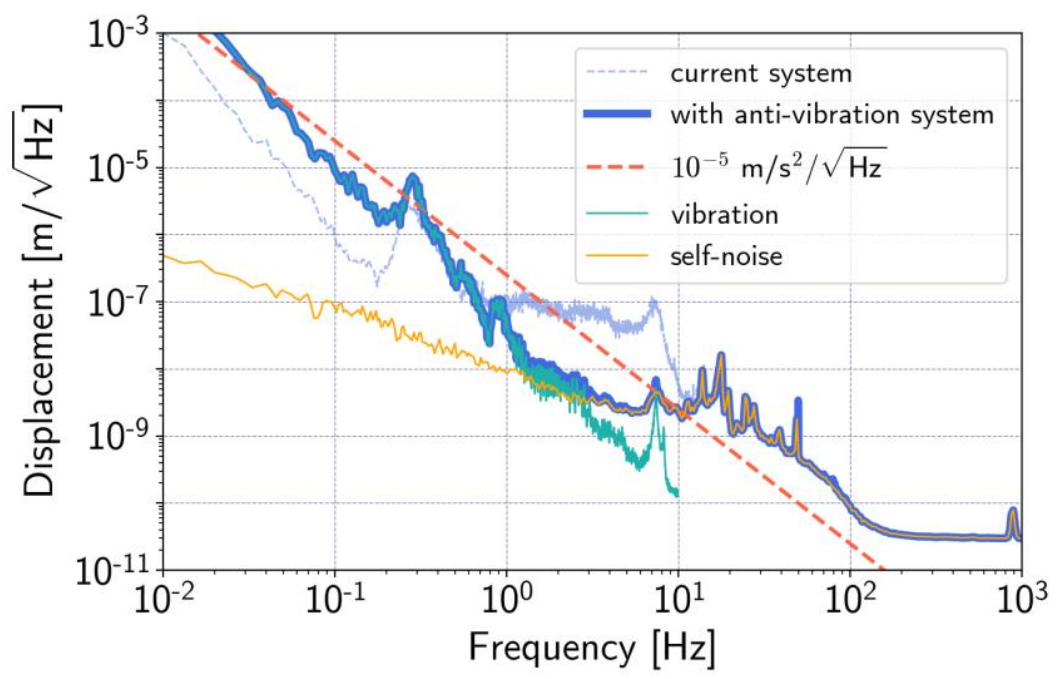

Figure 7: Expected improvement of the background noise of the calibration system by using the low-frequency antivibration system (thick blue). The contribution from the vibration of the interferometer stage (green) and from the selfnoise of the interferometer (orange) are also presented. For comparison, the current noise level is plotted with the dashed blue line.

\subsection{Expected Improvement of Interferometer Background Noise}

As explained in Section 2.1, the interferometer measures $\left(x_{\mathrm{a}}-x_{\mathrm{o}}\right)$, and $x_{\mathrm{o}}$ becomes background noise in accelerometer calibration. Therefore, total background noise of the interferometer can be estimated by the sum of the vibration spectrum $x_{\mathrm{o}}$ shown in Figure 6 and the interferometer self-noise spectrum shown in Figure 7.

Figure 7 presents the estimated noise level of the low-frequency accelerometer calibration system with the anti-vibration system. The noise is expected to be suppressed by a few tens of times between 1 and $10 \mathrm{~Hz}$, where the vibration of the interferometer stage is a dominant background noise. Such frequencies are important for the monitoring of infrastructure, which typically has a resonance frequency around $1 \mathrm{~Hz}$. In this frequency range, the noise level will be below $10^{-5} \mathrm{~m} / \mathrm{s}^{2} / \sqrt{\mathrm{Hz}}$. On the other hand, the self-noise of the interferometer dominates above $10 \mathrm{~Hz}$, hence the vibration isolation will not reduce the noise there. As mentioned in Section 2.1, suppression of the interferometer self-noise is required for further reduction of the background noise, especially above $10 \mathrm{~Hz}$. Alternatively, it will be easier to replace the interferometer to a low-noise commercial product to improve the calibration capacity.

\section{CONCLUSION}

An anti-vibration table is being installed into the low-frequency calibration system at NMIJ/AIST for low-acceleration measurement. The table has a low resonant frequency of $\sim 0.25 \mathrm{~Hz}$ to isolate the vibration at low frequencies. To evaluate the performance of the table, the vibration transmissibility was measured using a seismometer. The vibration was successfully isolated on the table by 100 times around a few $\mathrm{Hz}$. As a result, the interferometer background noise is expected to be lower than $10^{-5} \mathrm{~m} / \mathrm{s}^{2} / \sqrt{\mathrm{Hz}}$ below $10 \mathrm{~Hz}$, which enables the calibration system to determine the acceleration amplitude of $10^{-3} \mathrm{~m} / \mathrm{s}^{2}$ with $1 \%$ accuracy in a reasonable time. To extend the frequency range to above $10 \mathrm{~Hz}$, reduction of the interferometer noise other than the microtremor is necessary.

\section{ACKNOWLEDGEMENT}

This work is partially based on the results obtained from a project commissioned by the New Energy and Industrial Technology Development Organization (NEDO), Japan. The authors thank Tamio Ishigami, Koichiro Hattori, Akihiro Ota, Takashi Usuda, Hiromi Mitsumori, Yoshiteru Kusano (NMIJ) for useful discussions and cooperation.

\section{REFERENCES}

[1] K. Komatsu, H. Uchida, "Microvibration in spacecraft", Mechanical Engineering Reviews, Vol. 1, No. 2, 2014

[2] M. Privat, "On ground and in orbit microvibration measurement comparison", Proceedings of the $8^{\text {th }}$ European Conference on Spacecraft Structures, Material and Mechanical Testing, 1998

[3] D. Yu, G. Wang, Y. Zhao, "On-Orbit Measurement and Analysis of the Micro-vibration in a RemoteSensing Satellite". Adv. Astronaut. Sci. Technol. Vol. 1, pp. 191-195, 2018

[4] Y. Ikeda, S. Yoshitaka, S. Yasutsugu, "Damage detection of actual building structures through singular value decomposition of power spectral 
density matrices of microtremor responses", AIJ Journal of Technology, Vol. 16, No. 32, pp. 69-74, 2010 (In Japanese)

[5] Y. Jiang, Y. Gao, X. Wu, "The nature frequency identification of tunnel lining based on the microtremor method", Underground Space, Vol. 1, No. 2, pp. 108-113, 2016
[6] ISO 16063-11 1999 "Methods for the calibration of vibration and shock transducers-part 11: Primary vibration calibration by laser interferometry"

[7] W. Kokuyama, T. Ishigami, H. Nozato, A. Ota, "Improvement of very low-frequency primary vibration calibration system at NMIJ/AIST", in Proc. of XXI IMEKO World Congress, Prague, Czech Republic, 2015 